\title{
ELECTROCHEMICAL DETECTION OF NITROFURAL IN THE PRESENCE OF SODIUM AMMINEPENTACYANOFERRATE (II)
}

\author{
Iliyan Kolev', Sonya Ivanova ${ }^{2}$, Mihail Marinov ${ }^{3}$ \\ ${ }^{1}$ Department of Pharmaceutical Sciences and Pharmaceutical Management, \\ Faculty of Pharmacy, Medical University of Varna \\ ${ }^{2}$ Pharmacy „Poly“, POLIMEKS LTD, Varna \\ ${ }^{3}$ Freelance electronics engineer, Varna
}

\begin{abstract}
The present study deals with the development of a new qualitative electrochemical analytic test for detecting the chemotherapeutic agent Nitrofural. The combination of sodium amminepentacyanoferrate(II) and electric power, provided by a $9 \mathrm{~V}$ battery, was successfully implemented for the analysis of the medicine in question.
\end{abstract}

Keywords: pharmaceutical analysis, Nitrofural, nitro group, electrochemical reduction

\section{INTRODUCTION}

As a nonclassical bioisostere of the pyridine ring, the nitroaromatic structural motif may be found in a great number of artificial pharmaceuticals (Clonazepam, Flunitrazepam, Nitrazepam, Entacapone, Flutamide, Nifedipine, Nilutamide, Nifuroxazide, Nitrofurantoin, Metronidazole, etc., as well as the present examined Nitrofural medicine) $(1,2)$.

As a part of overall pharmacopoeial analysis, the qualitative control of nitroaromatic raw materials (medicines) is a task of paramount analytical importance. As a rule, the qualitative pharmacopoeial

Address for correspondence:

Iliyan Kolev

Faculty of Pharmacy

Medical University of Varna

84 Tzar Osvoboditel Blvd

9002 Varna

e-mail:ilian.kolev@mu-varna.bg

Received: June 7, 2018

Accepted: June 20, 2018

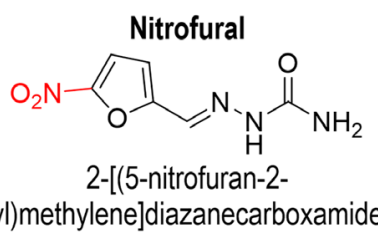

analysis is intended to confirm the identity of pharmaceutical raw materials at the so-assigned "acceptable" level of assurance (at the "second identification" stage). One of the positives of this analytical approach is its allowing a visual indication (analytical confirmation) of the chemical composition of the drug analyzed in toto or, partially of its peculiar structural motif (3-5). Therefore, in particular nowadays, the pharmacopoeial qualitative analysis is being widely used as a powerful analytical „instrument" for the identification of many drug raw materials, including the one examined here $(2,4)$.

The qualitative analysis of "nitro" medicines, ordinarily, proceeds via indirect tests on account of the chemical inertness of the $-\mathrm{NO}_{2}$ group, as distinct from the all remaining functional groups. This in- 
volves reactions of pyrolysis or catalytic reduction being run firstly (3).

In most cases (regardless of the type of aromatic residue), the modern qualitative pharmacopoeial analysis of $\mathrm{Ar}-\mathrm{NO}_{2}$ residues includes reduction transformations ${ }^{1}$ in which the analytes in question are preferentially converted to their much more reactive and analytically traceable nitroso, hydroxylamino, and amino forms or their azoxy and azo derivatives (Scheme 1). Being accompanied by a set of sophisticated methodical circumstances, however, the method of reduction analysis is difficult to execute. Also, the reduction tests are not so selective, inasmuch as a lot of other functional groups stated to give a false positive result (hydrazo-, azo-, azoxy- and nitroso-compounds) (6). cific manner viewing the applied potential, current density, and electrolytic solvents (7).

The electrochemical reduction of natural and synthetic nitro compounds has been drawing considerable attention for more than a century $(7,8)$. In the majority of scientific publications, the potential of those electrochemical (nitroreduction) methods has been evaluated in the context of their participation in the preparative organic chemistry, and in the remaining ones, in negligible volume, regarding their analytical application (8).

The electrochemical analysis of aromatic nitro compounds began early in 1934, when F. Feigl, V. Anger, and O. Frehden (9) accomplished qualitative electrochemical analysis of several artificial mono- and polynitro aromatic compounds in

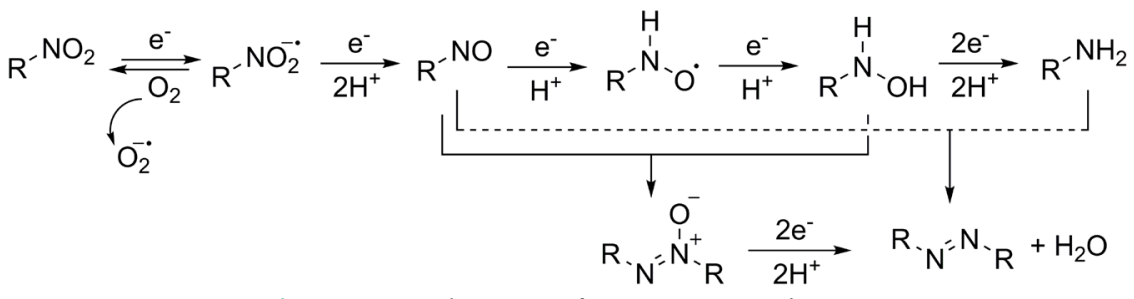

Scheme 1. Mechanism of nitro group reduction ${ }^{2}$

Sui generis, however, the reduction of the functional group in question may also be carried out electrolytically in the absence of any relatively expensive (and even toxic) reagents or promoters, even in a spe-

\footnotetext{
${ }^{1}$ The reduction of nitro groups may also be, in vivo, occurring as a result of metabolic transformations provoked by the action of cytochrome $\mathrm{P} 450$ in the presence of a competent redox partner (usually NADPH) (under anaerobic conditions) or the flavin-dependent NADPH cytochrome $\mathrm{P} 450$ reductase, as well as by bacterial nitro reductases (in the GI tract), and some hepatic oxidoreductases (1).

${ }^{2}$ As a whole, the mechanism of nitro reduction is very involved and strongly influenced by the nature (biological or artificial) of the reaction milieu.

${ }^{3}$ Many other nitrogen-containing functional groups besides the nitro ones may be involved in a contact with the sodium pentacyanoammineferroate, namely: hydrazines containing a free $\mathrm{NH}_{2}$ group, aromatic hydroxylamine compounds, nitroso compounds, and several amines including pyridine. A positive response to sodium pentacyanonitrosylferrate(II) has also been exhibited by thioketones, and some $\alpha, \beta$-unsaturated and aromatic aldehydes in the presence of hydrogen sulfide.
}

the presence of the titled inorganic reagent (sodium amminepentacyanoferrate(II) $\left.{ }^{3}\right)$. Shortly thereafter (10), however, the spot electrochemical test lost its practical significance, being substituted by new and much more fashionable analytical methods (11-15).

To complete the potential of Fiegl's electrochemical test in the field of the pharmaceutical analysis, a series of electroreduction spot tests including a pharmaceutical nitro representative Nitrofural and participation of a sodium amminepentacyanoferrate(II) reagent have been carried out. For the sake of operating simplicity, the main study objectives include: evaluation of the electrochemical behavior of the titled medicinal compound in the entourage of the inorganic Fe complex in question as well as establishing and optimizing of the experimental conditions for its qualitative electrochemical determination.

Furthermore, the present work may also be considered as a continuation of our earlier studies on the qualitative control (spot test analysis) of the bactericidal medicament Nitrofural (5). 


\section{MATERIALS AND METHODS}

All chemicals were of analytical grade and used as received from commercial sources: sodium nitroprusside $^{4}$ (p.a., Chem-Lab), Nitrofural (98+\%, Alfa Aesar), Nitrofurantoin (98+\%), Chloramphenicol (98\%, Acros Organics), Metronidazole (99\%, Acros Organics), 4-nitrotoluene (99\%, Sigma-Aldrich), ethyl 4-nitrobenzoate ( $\geq 98 \%$, Sigma-Aldrich), 4-nitrobenzoic acid (99\%, Alfa Aesar), 4-nitrobenzaldehyde (98\%, Sigma-Aldrich), sodium acetate anhydrous ( $\geq 99.0 \%$, Chem-Lab). The solvents ethanol (anhydrous), DMSO, and water were received from Fisher Scientific. All reactions were performed under ambient conditions using standard glass depression slides.

Synthesis of sodium amminepentacyanoferrate (II) $)^{5}$ : A $250 \mathrm{ml}$ pyrex screw-capped reagent bottle was charged with $10 \mathrm{~g}$ of sodium nitroprusside, $6.0 \mathrm{~g}$ of sodium acetate and $75 \mathrm{ml}$ of concentrated $\mathrm{NH}_{4} \mathrm{OH}$. After keeping the mixture below $5^{\circ} \mathrm{C}$ overnight (in a refrigerator), the bright yellow crystals formed were filtered off by suction. Then, the residue was dissolved in a minimum amount of cold water and reprecipitated with a minimum amount of precooled ethanol. After filtration, the solid was washed repeatedly with $5.0 \mathrm{ml}$ of cold ethanol and dried under a stream of dry nitrogen (for about of 2 hours). An insignificant quantity of the so-obtained crude product, marked as $\mathrm{Na}_{3}\left[\mathrm{Fe}(\mathrm{CN})_{5} \mathrm{NH}_{3}\right] \cdot \mathrm{xH}_{2} \mathrm{O}$, was used for the preparation of its $1 \%$ aqueous solution.

Experimental procedure: Each glass depression was loaded with a drop of a freshly prepared solution of $1 \%$ sodium amminepentacyanoferrate and a drop of $1 \%$ solution of Nitrofural in DMSO ${ }^{7}$. Then the current, drawn from a flashlight battery, was passed for at least $5 \mathrm{~min}$ through the so-obtained mixture ${ }^{2}$.

${ }^{4}$ Synonyms: Sodium pentacyanonitrosoferrate(III), Nipride, Nitropress, used also as a medication in cases of acute hypertensive crises.

${ }^{5}$ The synthetic procedure developed by D. J. Kenney et al. has been successfully employed (16) for this purpose.

${ }^{6} 1 \%$ solutions of Nitrofurantoin, Chloramphenicol, and Metronidazole in DMSO were also prepared.

${ }^{7}$ The graphite sticks $(0.7 \mathrm{~mm}$ in diameter, immersed 1.0 $\mathrm{mm}$ into the test solution, resulting in a working electrode surface of about $5.1 \mathrm{~mm} 2$ and a current density of $39.2 \mu \mathrm{A} / \mathrm{mm} 2$ ) were spaced $4.0 \mathrm{~mm}$ apart. The anode and the cathode sticks were clamped parallel to each other and connected to the current source by means of "croco-

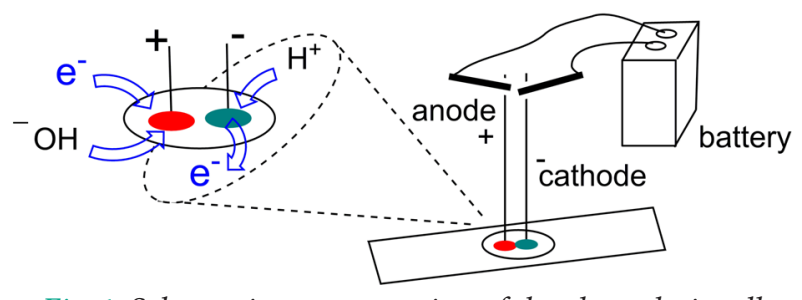

Fig. 1. Schematic representation of the electrolysis cell used for the analysis of chemotherapeutic agent Nitrofural

Development of red and greenish-blue colored zones during the electrolysis should be observed.

All tests were repeated three times to confirm the repeatability of the electrochemical analysis.

Caution!!! All work with ammonia and DMSO must be performed under a well-ventilated hood using protective clothing and gloves.

\section{RESULTS AND DISCUSSION}

Microdetection or the so-called "spot test" analysis of aromatic and heterocyclic nitro compounds has been the subject of continuing analytical interest over the last several decades, owing to the steadily increasing number of nitrogen-containing medicines, polymers, production intermediates, and artificial raw materials. Up to now, the implementation of various analytical methods in the qualitative analysis of aromatic nitro compounds has been reported on in the scientific periodicals. In some of them the analysis of nitro compounds has been performed by their fusion with diphenylamine or N,N-diethylaniline (17) or through their pyrolytic-reductive "lysis" to nitrous acid $(3,18)$, as well as through their catalytic reduction to the highly reactive and easily traceable hydroxylamine and nitroso forms, or through their saponification to the analytically identifiable nitrite salts (3). In other papers the qualitative analysis of nitro compounds has been indirectly accomplished by additional, consistently applied, and complicated analytical reactions (19-21).

As to the electrochemical method developed by Feigl et. al. (9), it should once again be emphasized on due to its originality, effectiveness, and efficiency in the qualitative analysis of artificial aromatic nitro compounds. The method is unique both by its experimental design, and by its reactant specificity. Furthermore, the methodology of the test allows its easy

dile" clips and electrical wires, as shown in Figure 1. 


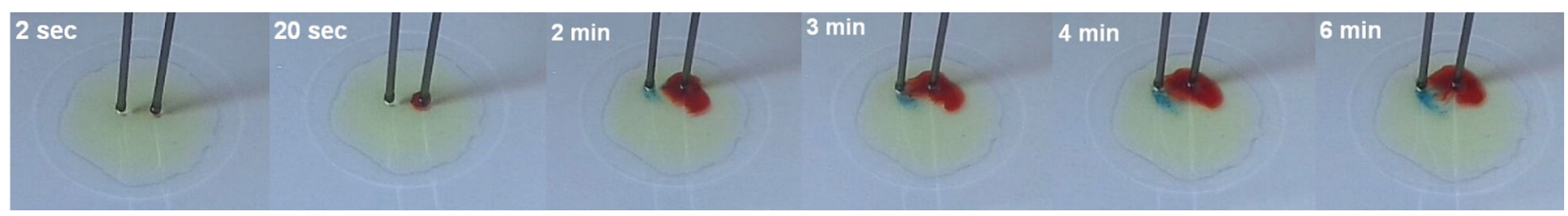

Fig. 2. A photograph illustrating the appearance of two different colored zones in the space around the working electrodes

adaptation and ubiquitous application in the qualitative organic analysis.

In order to assess the potential of this electrochemical method in the scope of pharmaceutical analysis we have initiated a series of tests for investigating the reactivity of the chemotherapeutic agent Nitrofural in the presence of sodium amminepentacyanoferrate.

Actually, as expected, a positive electroanalytical response was observed when an intermittent current, drawn from a $9 \mathrm{~V}$ battery, passed through the tested solution. Surprisingly, however, the electrochemical reduction of Nitrofural led to the formation of two clearly colored individual zones in the vicinity of both working electrodes, namely: a red colored spot surrounding the anodic surface and a greenishblue colored one about the cathodic vicinity (Fig. 2).

The appearance of a red stain has been associated with the rapid and localized $\mathrm{pH}$ changes in the composition of the reaction medium during its electrolysis; with changes, which bring about the formation of a violet-red colored Nitrofual nitronato-quinone form.<smiles>NC(=O)N=N/C=C1\C=CC(=[N+]([O-])[O-])O1</smiles>

Analogous alterations in the coloration of the Nitrofual DMSO solution were also registered during its mild and intentional alkalization with $0.1 \mathrm{M}$ $\mathrm{NaOH}(2)$.

The local changes in $\mathrm{pH}$ of the electrolytic solution were successfully proven and visualized with the aid of a moistened indicator paper (part of strip) embedded in the groove of the used glass slide (Fig. 3).

In this connection, for comparison, the electrochemical behavior of the remaining compounds examined is also shown. Despite the structural similarities, as expected, no other mononitro-containing compound, including Nitrofurantoin analog, manifested similar anodic behavior - a behavior, which

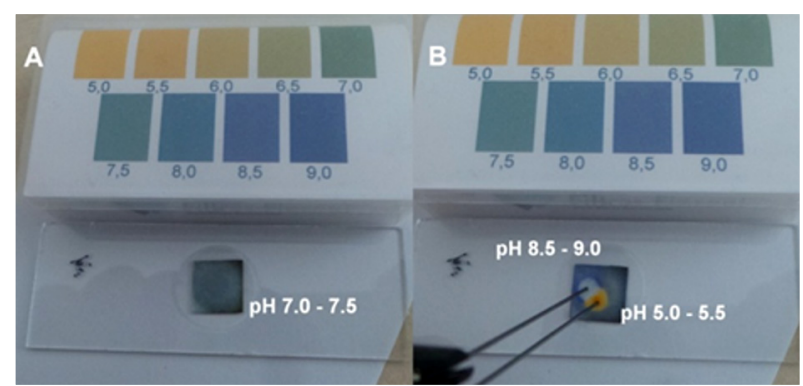

Fig. 3. Photograph illustrating the differences in the values of $\mathrm{pH}$ in the peri-electrodic spaces obtained during the electrolysis process

should emphasize the specificity of the present electrochemical method towards the tested nitrofuranoic medicine.

The electrochemical changes in the Nitrofural analyte about the cathodic surface have been demonstrated in an irrefutable way by the reductive transformation of its nitro functional to a reactive intermediate, which is capable of dyeing the used $\mathrm{Na}_{3}\left[\mathrm{Fe}(\mathrm{CN})_{5} \mathrm{NH}_{3}\right]$ complex (Fig. 4). Here, complex-formation reactions between the inorganic salt used (which is also stated as an effective complexing agent) and in situ generated reduced Nitrofural forms would be realized $(9,16)$.

In order to justify the above-proposed mechanism and exclude a possible presence of false-positive reactions, additional tests were performed with respect to all reactant species utilized.

As expected, none of the reagents used, have been shown to give such a bicolor "positive" result. A negative response has also been detected even at the electrolysis of larger amounts of the $\mathrm{Na}_{3}\left[\mathrm{Fe}(\mathrm{CN})_{5} \mathrm{NH}_{3}\right]$ salt used, where oxidation-reduction reactions associated with decomposition of the of the pentacyanamine complex and the formation of Prussian blue salt may have taken place (16).

Moreover, the negative controls give also evidence that none of the medicines used has been contaminated with their partially reduced nitro forms forms, which can occur as a result of their partial bi- 


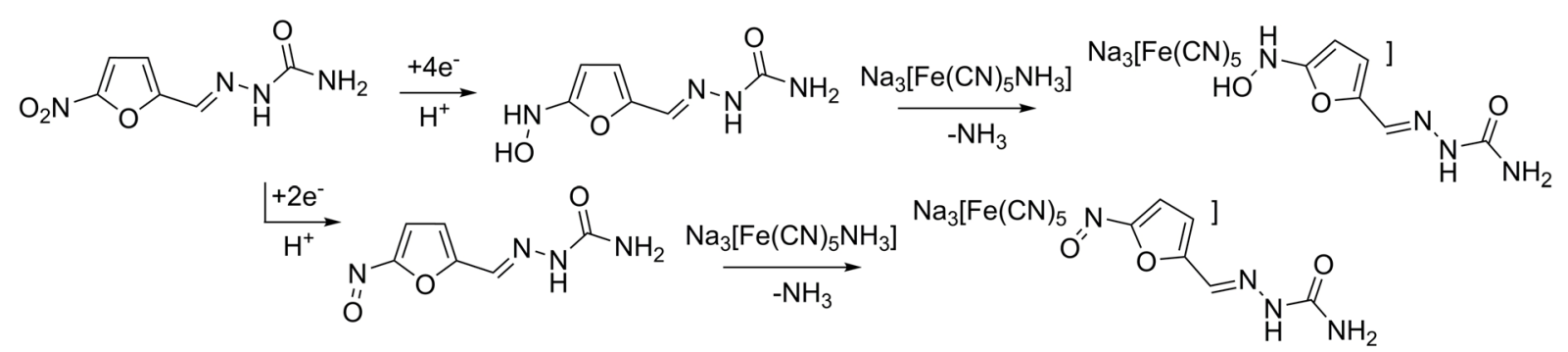

Fig. 4. Proposed mechanism of the interaction of $\mathrm{Na}_{3}\left[\mathrm{Fe}(\mathrm{CN})_{5} \mathrm{NH}_{3}\right]$ reagent with the Nitrofural chemotherapeutic

ological contamination during sampling or erroneous storage (see Footnote 1).

\section{CONCLUSION}

The present article describes a simple, rapid, and highly specific electrochemical method for detection of the chemotherapeutic agent Nitrofural in the presence of sodium amminepentacyanoferrate (II). In a series of experiments, the proposed electrocolorimetric test has proven its reliability and efficiency in the qualitative analysis of the medicine in question. The presented method differs from the existing spot test approaches by being completely defined by a set of experimental circumstances, namely - by the formation of two clearly colored distinct zones in the vicinity of the working electrodes; a red colored spot around the anodic surface and a greenish-blue colored one about the cathodic area.

Although simple, the method presented appears to be more reliable and specific regarding the used Nitrofural analyte being indifferent towards all of the remaining tested nitro compounds (Nitrofurantoin, Chloramphenicol, Metronidazole, 4-Nitrotoluene, Ethyl 4-nitrobenzoate, 4-Nitrobenzoic acid, and 4-Nitrobenzaldehyde).

Moreover, it requires the usage of only one inorganic reagent, $\mathrm{Na}_{3}\left[\mathrm{Fe}(\mathrm{CN})_{5} \mathrm{NH}_{3}\right]$, which can either be obtained easily or purchased.

Being accurate, reproducible, and specific, the presented method seems to be completely applicable and suitable for the routine pharmaceutical quality control (second identification analysis) of the raw drug substance Nitrofural.

\section{REFERENCES}

1. Silverman R; Holladay M. The organic chemistry of drug design and drug action. Amsterdam: Elsevier; 2014.
2. European Pharmacopoeia, Strasbourg: Council of Europe. 8th ed., vol. 1 and 2; 2013.

3. Feigl F. Spot Tests In Organic Analysis. Amsterdam: Elsevier; 1966.

4. Pedersen O. Pharmaceutical Chemical Analysis: Methods for Identification and Limit Tests. Boca Raton: Taylor \& Francis Group; 2006.

5. Kolev IN, Ivanova SJ. The unusual behavior of Gibbs' reagent versus Nitrofural. Scr Sci Pharm. 2018;5(1): 7-12. DOI: http://dx.doi.org/10.14748/ssp. vli1.4996.

6. Bose PK. A New Method for the Detection of the Nitro-Group in some Organic Compounds. Analyst. 1931;56(665):504-7. DOI: 10.1039/ AN9315600504.

7. Fuchigami T, Atobe M, Inagi S. Fundamentals and Applications of Organic Electrochemistry: Synthesis, Materials, Devices. Chichester: John Wiley \& Sons; 2014.

8. Hammerich O; Speiser B. (eds.) Organic Electrochemistry. 5th ed. Boca Raton: Taylor \& Francis Group; 2016.

9. Feigl F, Anger V, Frehden O. Über die Verwendung von Tüpfelreaktionen zum Nachweis von organischen Verbindungen. (IV.) Mikrochemie. 1934;15(1):181-9. DOI: 10.1007/bf02789367.

10. Ohkuma S. A microdetection of aromatic nitro compounds with sodium pentacyanoammineferroate. YAKUGAKU ZASSHI. 1955;75(11):1342-45. DOI: 10.1248/yakushi1947.75.11_1342.

11. Barek J, Cvačka J, Muck A, Quaiserová V, Zima J. Polarographic and Voltammetric Determination of Carcinogenic Nitro and Amino Derivatives of Polycyclic Aromatic Hydrocarbons. Electroanalysis. 2001;13(8-9):799-803.

12. Mishra AK, Gode KD. Electrochemical reduction of nitrofurazone and its determination in pharmaceutical dosage forms by d.c. polarogra- 
phy. Analyst. 1985;110(11):1373-6. DOI: 10.1039/ an9851001373.

13. Reday CS, Reddy SJ. Electrochemical reduction of nitrofurazone and its determination in pharmaceutical dosage forms by differential pulse polarography. Electroanalysis. 1992;4(5):595-9. DOI: 10.1002/elan.1140040515.

14. Hirokazu T, Kazuo M, Keiko T, Saburo N. Fluorometric analysis with o-Aminothiophenol. V. Fluorometric determination of Nitrofurazone. YAKUGAKU ZASSHI. 1974;94(6):717-23. DOI: 10.1248/yakushi1947.94.6_717.

15. Guo X, Wang Y, Wu F, Ni Y, Kokot S. The use of tungsten disulfide dots as highly selective, fluorescent probes for analysis of nitrofurazone. Talanta. 2015;144:1036-43. DOI: 10.1016/j. talanta.2015.07.055.

16. Beckman HF. Feed Additives Analysis, Microdetermination of the Medicaments Furazolidone and Nitrofurazone. J Agric Food Chem. 1958;6(2):1302. DOI: $10.1021 /$ jf60084a009.
17. Verma KK, Dubey SK. Detection of aromatic nitro compounds by means of their $\pi$-complexes with N,N-diethylaniline. Talanta 1981;28(7):485-6. DOI: 10.1016/0039-9140(81)80074-4.

18. Feigl F. Spot test detection of nitrocellulose. Chemist-Analyst. 1963;52:47.

19. Jungreis E, Lipetz V. Sensitive and Selective Spot Test for m-Amino- and m-Nitro-Phenol. Microchim Acta. 1963;51(5-6):886-8. DOI: 10.1007/ bf01217613.

20. Basu K, Chakraborty S, Saha C. A closer look at the Mulliken-Barker test. Resonance. 2013;18(9):854-8. DOI: 10.1007/s12045-013-0108-1.

21. Hearon WM, Gustavson RG. A semimicro qualitative test for the nitro group in organic compounds. Ind Eng Chem Anal Ed. 1937;9(7):352-3. DOI: 10.1021/ac50111a024. 